\title{
Multiphase Flow in the Gap Between Two Rotating Cylinders
}

\author{
Milada Kozubková ${ }^{1, *}$, Jana Jablonská ${ }^{1}$, Marian Bojko ${ }^{1}$, František Pochylý2 ${ }^{2}$, and Simona \\ Fialová ${ }^{2}$ \\ ${ }^{1}$ VSB - Technical University of Ostrava, Faculty of Mechanical Engineering, Department of \\ Hydromechanics and Hydraulic Equipment, 17. listopadu 2172/15, 70800 Ostrava-Poruba, Czech \\ Republic \\ ${ }^{2}$ BUT - Brno University of Technology, Faculty of Mechanical Engineering, Victor Kaplan Dept. of \\ Fluid Engineering, Technická 2896/2, 61669 Brno, Czech Republic
}

\begin{abstract}
The research of liquids composed of two (or more) mutually immiscible components is a new emerging area. These liquids represent new materials, which can be utilized as lubricants, liquid seals or as fluid media in biomechanical devices. The investigation of the problem of immiscible liquids started some years ago and soon it was evident that it will have a great application potential. Recently, there has been an effort to use ferromagnetic or magnetorheological fluids in the construction of dumpers or journal bearings. Their advantage is a significant change in dynamic viscosity depending on magnetic induction. In combination with immiscible liquids, qualitatively new liquids can be developed for future technologies. In our case, immiscible fluids increase the dynamic properties of the journal hydrodynamic bearing. The article focuses on the stability of single-phase and subsequently multiphase flow of liquids in the gap between two concentric cylinders, one of which rotates. The aim of the analysis was to study the effect of viscosity and density on the stability/instability of the flow, which is manifested by Taylor vortices. Methods of experimental and mathematical analysis were used for the analysis in order to verify mathematical models of laminar and turbulent flow of immiscible liquids.
\end{abstract}

\section{Introduction to the issue}

The immiscible liquids in question consist of two (or more) liquid and/or solid components (liquid-liquid or liquid-solid phases). Their behaviour depend on their chemical composition, physical properties, and on influence of external factors like a magnetic field. The solid phase is represented by the particles dispersed in a carrying fluid. Their interaction with the outer environment is effected by their chemical substance, surface coating, and can be influenced by external fields. Due to the effect of the magnetic field, liquids already become nonNewtonian $[6,7,8]$. The following article is focused on the research of Newtonian fluids, where the basic flow characteristics are verified on the basis of computational modeling and

\footnotetext{
*Corresponding author: milada.kozubkova@,vsb.cz
} 
experiment. Due to the fact that flow instabilities penetrate at the interface of immiscible liquids, we decided to study the behavior of immiscible liquids in the area where another type of instability manifests itself, ie Taylor's vortex, because this issue is described in sufficient detail in $[1,2,3,4,5]$.

Taylor vortices are instabilities arising when a critical Taylor number is reached, which depends, among other things, on the rotational speed of the inner resp. outer cylinder and fluid viscosity. By gradually increasing the rotational speed, different Taylor-Couett flow modes can be achieved, see Figure 1, [1,2].
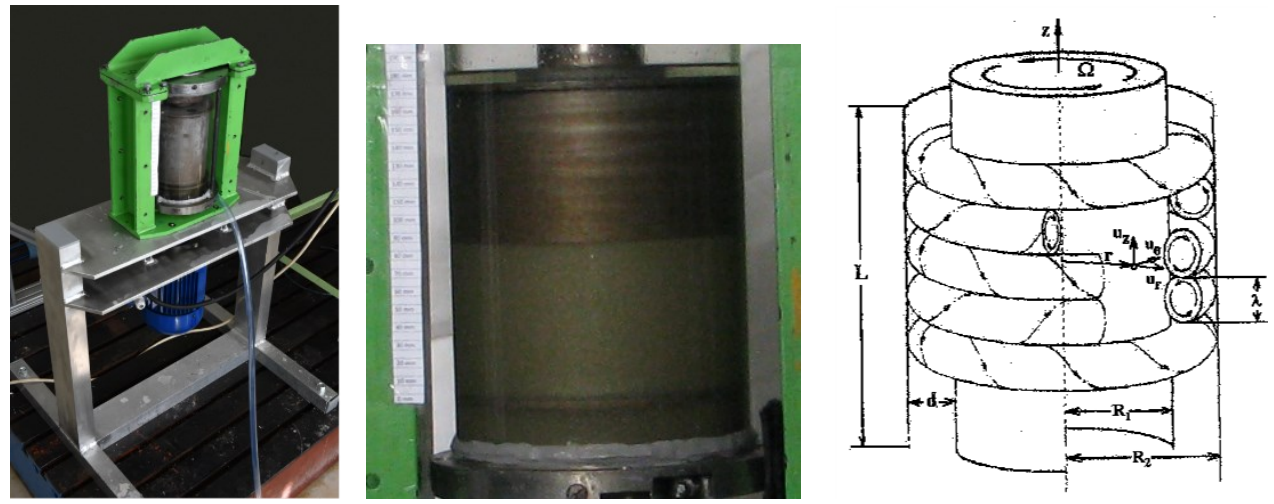

Fig. 1. Flow characteristics between concentric cylinders during rotation of the inner cylinder, photo of the experiment, detail, scheme of the area.

The flow type is determined from the geometry, operating parameters and subsequently dimensionless numbers, such as the Reynolds number, the value of which determines the type of flow (laminar vs. turbulent) and the Taylor number, which determines whether Taylor vortices occur in this gap between cylinders at zero pressure. slope. The axial pressure drop suppresses the existence of these vortices. The definition of these parameters in the case of flow between rotating cylinders is as follows.

The Reynolds number for this configuration is given:

$$
\operatorname{Re}=\frac{\Omega R_{1}\left(R_{2}-R_{1}\right)}{v}
$$

where $\Omega$ is the angular velocity, $R_{l}$ is the inner radius, $R_{2}$ is the outer radius, $v$ is the kinematic viscosity. The critical value of the transition from laminar to turbulent flow is in the interval $\langle 1100-1400\rangle$.

Taylor numberis defined as:

$$
\mathrm{Ta}=\operatorname{Re} \sqrt{\frac{R_{2}-R_{1}}{R_{1}}}=\frac{\Omega R_{1} d}{v} \sqrt{\frac{R_{2}-R_{1}}{R_{1}}}
$$

where $d=R_{2}-R_{1}$. The critical value characterizing the formation of stationary vortices is 41.3 and the periodic wave modes of vortices are up to one hundred times critical Taylor number, see Figure 1.

An experimental method can be used to investigate the multiphase flow of liquids. For this reason, the flow of oil between rotating concentric cylinders was solved to create vortex structures called Taylor vortices. The problem of stability analysis has been investigated at the workplace in the past [1]. In the same experimental device, other liquids with different physical properties and subsequently a mixture of these liquids were tested. 


\section{Experiment, physical properties of fluids, Re and Ta number}

Flow visualization is a very suitable tool for experimental fluid mechanics and is necessary in the study of complex flow cases. A very interesting case there are the flow structures in connection with the Taylor-Couette flow, defined by the flow of a viscous liquid in an annulus between two cylindrical surfaces which move relative to each other.

The basis of the measuring device (see Figure 1) is a stable supporting construction with an electric motor enabling to reach the maximum rotational speed of the inner cylinder. The electric motor is controlled by a frequency converter. The upper part of the device is fitted with its own measuring device consisting of two concentric cylinders. The outer cylinder is made of glass, the inner cylinder is steel. It was also necessary to eliminate all vibrations in the device, which could result in the destruction of the entire system at higher rotational speeds.

The parameters of the device are as follows:

$$
\begin{aligned}
& R_{l}=65 \mathrm{~mm} \text {............. inner cylinder radius } \\
& R_{2}=80 \mathrm{~mm} \text {............. outer cylinder radius } \\
& d=15 \mathrm{~mm} \text {............. thickness of annulus }\left(R_{2}-R 1\right) \\
& L=170 \mathrm{~mm} \text {................ length of cylinder }
\end{aligned}
$$

With the help of aluminum powder, the individual limit states of Taylor vortices can be recognized and the rotational speed ranges in which they are located can be recorded. There is also a change in the number of bands that characterize the number of vortices created.

\subsection{Physical properties of liquids}

Commonly available liquids or their mixtures were tested in the experimental equipment described above, see Table 1. The transition state and vortex structures are best observed with hydraulic oil using aluminum powder. The change in viscosity with the addition of aluminum sawdust was negligible by about $2 \%$ [1]. Taylor vortices in water are only observable at very low rotational speeds, but visibility by aluminum powder is impossible, so alcohol was used in terms of visibility.

Table 1. Physical properties of liquids.

\begin{tabular}{|c|c|c|c|c|c|}
\hline & units & water & alcohol & oil & $\begin{array}{c}\text { oil + alcohol } \\
\mathbf{1 : 1}\end{array}$ \\
\hline Density & $\mathrm{kg} \cdot \mathrm{m}^{-3}$ & 998 & 790 & 876 & 833 \\
\hline Kinematic viscosity & $\mathrm{m}^{2} . \mathrm{s}^{-1}$ & $1.002 .10^{-6}$ & $1.5209 .10^{-6}$ & $8.2182 .10^{-5}$ & $4.3937 .10^{-5}$ \\
\hline Dynamic viscosity & $\mathrm{Pa} . \mathrm{s}$ & 0.001 & 0.0012 & 0.072 & 0.0366 \\
\hline Surface tension & $\mathrm{N} . \mathrm{m}^{-1}$ & 0.073 & 0.0226 & 0.030 & estimation 0.011 \\
\hline
\end{tabular}

\subsection{Taylor and Reynolds number}

The type of flow and the origin of Taylor vortices are determined by the values of the Reynolds and Taylor numbers. The laminar resp. Turbulentn flow regime is defined by the critical value of the Reynolds number for flow in a narrow gap $\left(\operatorname{Re}_{\text {crit }}=1100\right.$ to 1300). Taylor's vortices appear after exceeding Taylor's critical number $\left(T a_{c r i t}=41.3\right)$. There are 
other variants of these vortices (wave mode, spiral mode, etc.) where it is difficult to determine the limit values of the Taylor number.

From the above definitions of Reynolds and Taylor number, the dependences of Reylolds and Taylor number on the rotational speed of the inner cylinder and the type of liquid, ie viscosity, were constructed, see Figure 2 . In addition, the graphs show the values of critical Taylor number and critical Reynolds number.
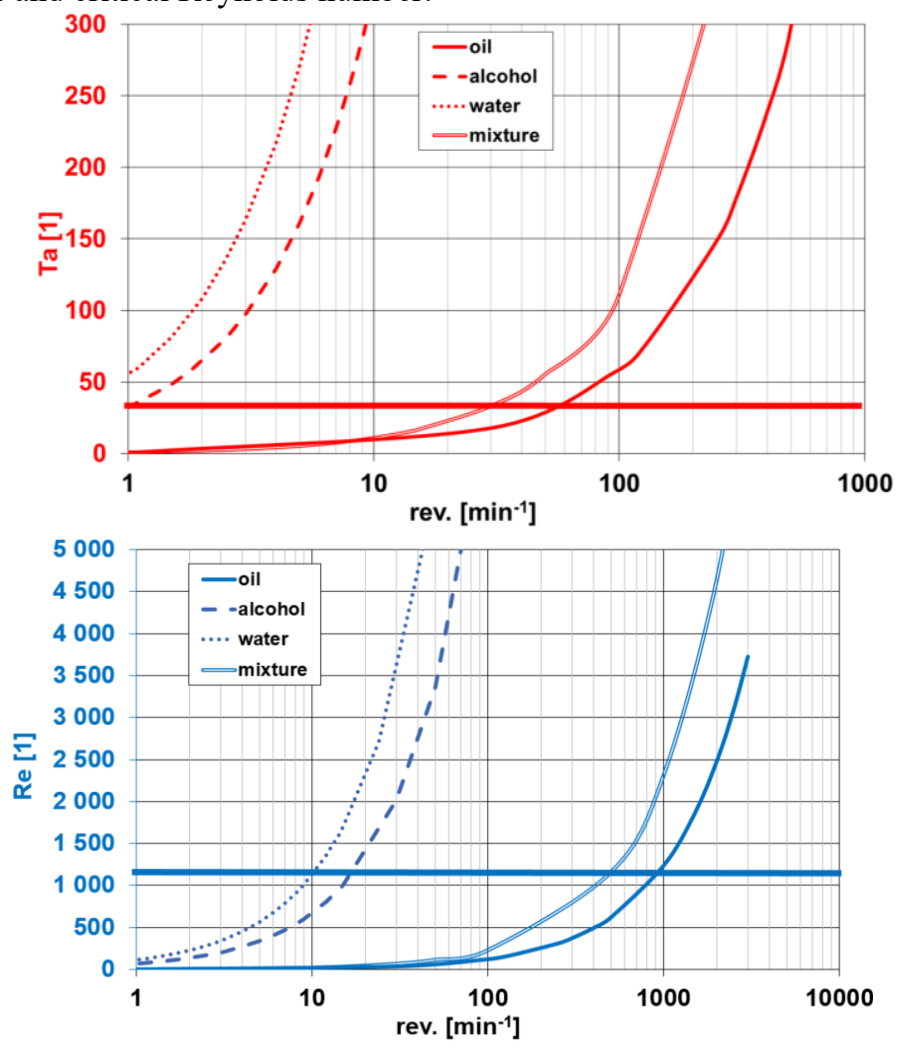

Fig. 2. Dependence of Taylor and Reynolds number on rotational speed $\left(T a_{c r i t}=41.3, R e_{c r i t}=1100\right.$ to 1300).

It is evident that the flow is located in the laminar region, in the transition region between laminar and turbulent flow and in the turbulent region due to the possibilities of the experiment. From the graph Re vs. rotational speed it is clear that water and alcohol flow at minimum rotational speed already in the turbulent mode or transition from laminarity to turbulence, while the flow of oil and mixture is laminar and changes to turbulent up at rotational speed values of the order of $1000 \mathrm{~min}^{-1}$. From the graph Ta vs. Rotational speed it is evident that for water and alcohol it is possible to expect the formation of vortices practically at the minimum rotational speed that the physical device is able to realize. For oil and oil-alcohol mixtures, Taylor vortices occur at approximately $100 \mathrm{~min}^{-1}$. This information will be used to define a mathematical flow model.

\section{Evaluation of experimental measurements}

Hydraulic oil and alcohol were used to visualize the single-phase flow. Alcohol shows similar properties, only the critical rotational speed is very small. In the experiment it is not possible to change such low rotational speed continuously, the lowest is approximately $10 \mathrm{~min}^{-1}$. The 
number of vortices is influenced by the boundary conditions and the specific liquid and will take values of approximately 10 (even number), which is confirmed by the experiment. This statement will also be used to verify the numerical experiment.

In the case of multiphase flow, experience with the flow of single-phase liquids has been used. A number of experiments were performed where oil and alcohol were used in different volume ratios. At low rotational speeds, no homogeneous mixture was formed, the higher density oil was placed at the bottom of the annulus and the lower density alcohol at the top of the annulus. At increasing rotational speeds, the liquids began to change the observable flow structure, mix and subsequently form a mixture. The best observable flow structure was present at a volume fraction of these liquids of 1:1. Table 2 shows one example of results of the alcohol, oil and a mixture flow for rotational speed 10. Further results are used in Table 3 , where they were also compared with numerical results.

Table 2. Flow visualization for $10 \mathrm{~min}^{-1}$ rotational speed.

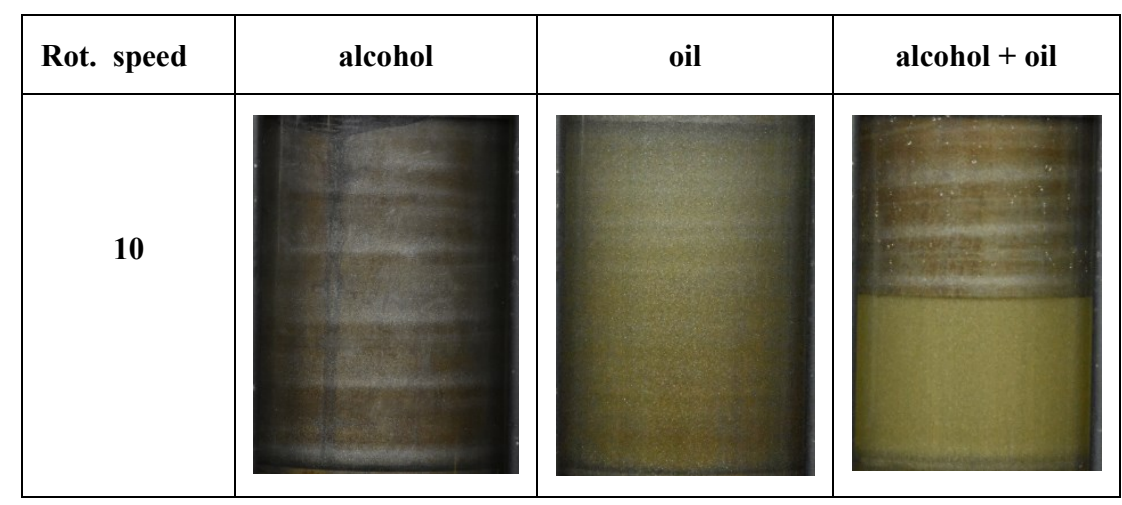

The following differences could be observed from the experiment due to significantly different physical properties, especially viscosity:

- Taylor vortices can be observed mainly in the region of laminar flow, therefore in alcohol they appear at low rotational speeds, on the contrary in oil vortex structures there are formed at rotational speed of 700 .

- Turbulence in alcohol causes vortex structures to be illegible.

- Similar behavior can be observed for multiphase flow of two liquids until turbulence begins to act significantly and a mixture of two separate phases is formed.

\section{Evaluation of numerical simulation}

In single-phase flow, the problems of oil and alcohol flow were tentatively solved, in twophase flow, a mixture of oil and alcohol in a volume ratio of 1:1. The geometry is axially symmetric, so the problem was solved in $2 \mathrm{D}$. The axis of rotation was located in the vertical direction, so the gravitational force was considered in the calculation. The single-phase flow of oil and alcohol was solved in accordance with previous applications, ie a laminar model was used for the laminar mode, a turbulent model for the Spalart Allmaras turbulent mode, both in the finite volume method. Physical properties were given according to Table 1. The boundary conditions are very simple, i.e. the rotational speed has been defined for a cylinder with a small radius and for attachments closing the cylindrical gap from below and above and zero rotational speed for the outer transparent cylinder. Due to the experimental results, when there was no significant wave mode, a simplified 2D geometry was used. When solving the two-phase flow, the oil with a higher density was established in the lower part of the annulus and the alcohol in the upper part. When rotating in the laminar mode, the liquids did 
not mix even with the formation of Taylor vortices (rotational speed 10, $30 \mathrm{~min}^{-1}$ ). During the transition to the turbulent mode, the liquids were mixed and vortices of the same size were established along the entire length. Density and viscosity is determined by the formula for the mixture. Both Mixture and Euler-Euler multiphase model gave the same results [9].

Table 3. Rotational speed-dependent flow simulation and comparison with experiment.

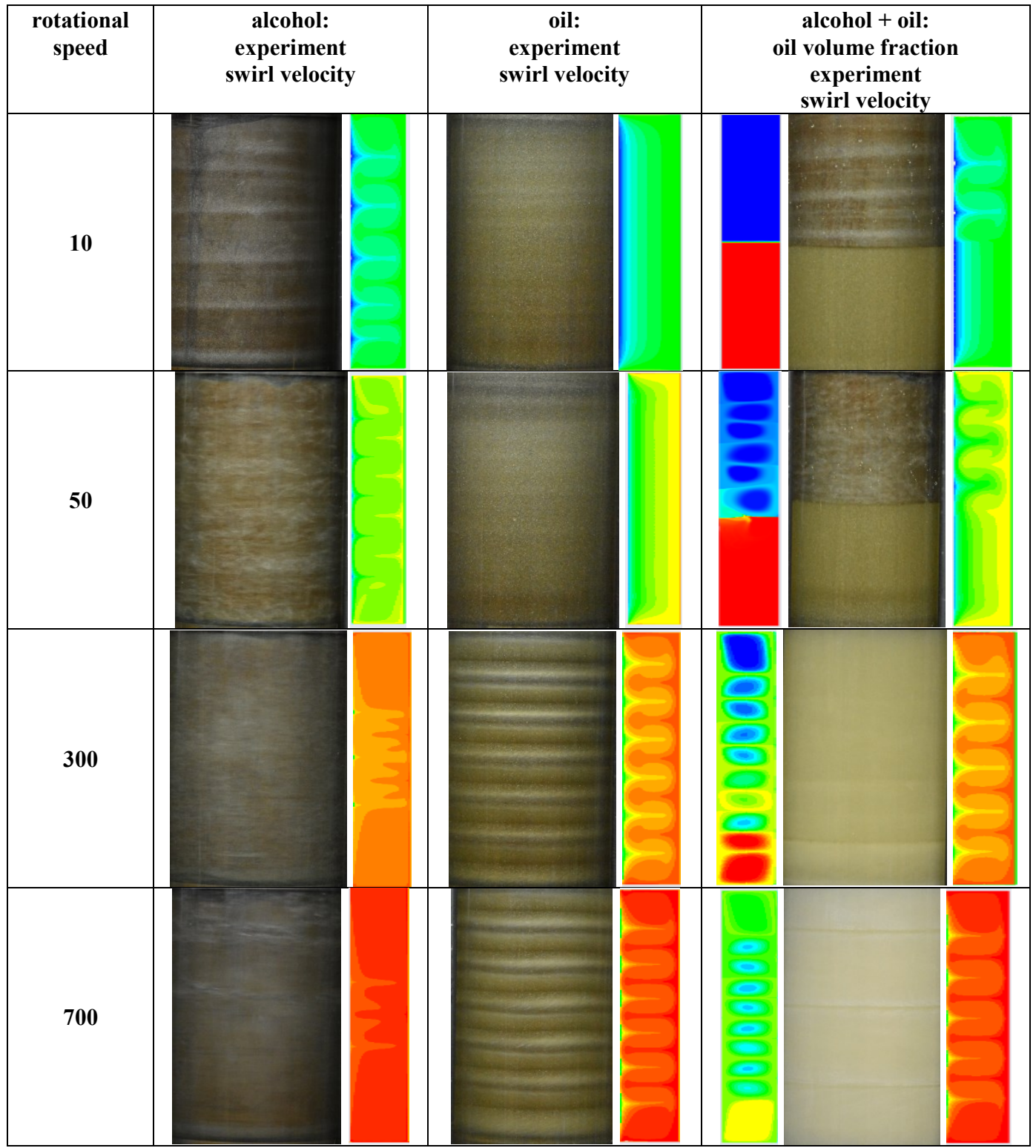

It can be seen from the table that the swirl velocity of single-phase and two-phase flow is practically the same. The distribution of oil and alcohol in two-phase flow is interesting. At rotational speeds up to 700 , it can be stated that there is no complete mixing of the liquid, which is not entirely clear in the visualization. However, the liquid is well mixed near the walls of both cylinders, so we can talk about emulsion at the walls.

Figure 3 evaluates the swirl velocity profile in logarithmic coordinates depending on the liquid (alcohol, oil, mixture) and the number of revolutions. The number of tapes in the 
experiment can be determined by the number of rotational speed peaks. Of interest is the asymmetrical evolution of the rotational speed for the mixture at low rotational speeds, where part of the alcohol and part of the oil can be distinguished.

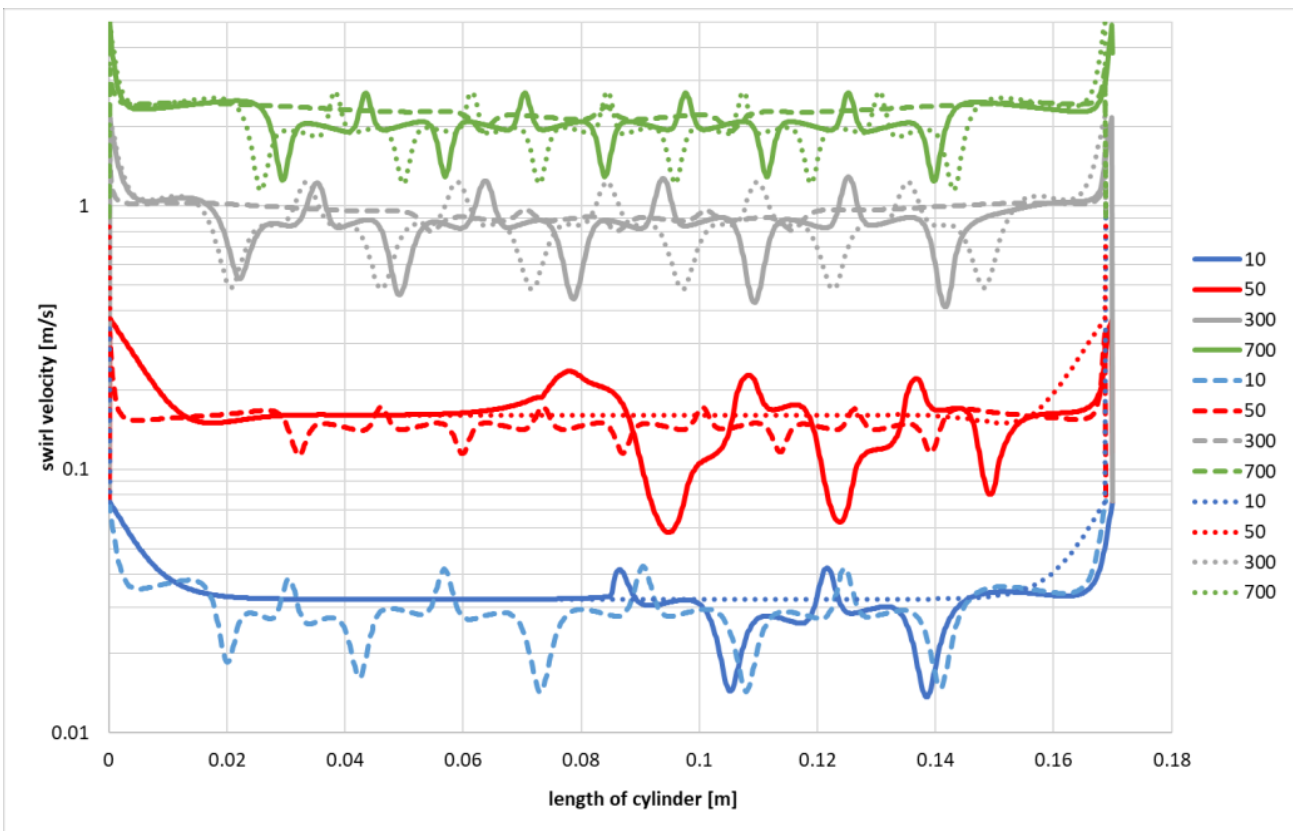

Fig. 3. Dependence of swirl velocity in logarithmic coordinates on the length of the cylinder. .... oil ---- alcohol — mixture

\section{Conclusion}

The aim of the work was to verify how single-phase and two-phase fluid behaves when flowing in the annulus, where the inner cylinder rotates. The gravitational force and the position of the axis of rotation are respected.

- The flow of a single-phase fluid and vortex structure (Taylor vortices) can be characterized by Taylor and Reynolds numbers.

- The flow of a two-phase liquid at low rotational speed does not form a mixture, while each phase is characterized by the respective Taylor and Reynolds number.

- The flow of two-phase liquid at higher rotational speeds begins to form a mixture, but inhomogeneous. Near the walls of the cylinders the mixture is homogeneous, but in the middle it is possible to distinguish partially individual liquids.

- The mathematical model differs:

- oil - laminar flow model

- alcohol - for rotational speeds of 10 and $30 \mathrm{~min}^{-1}$ a laminar flow model is used, for higher rotational speeds a turbulent flow model is used

- alcohol + oil - if the rotational speed is 10 and $30 \mathrm{~min}^{-1}$, the mathematical model used is laminar, for higher rotational speeds it is turbulent, because the phases are not strictly separated.

The modeling results are in agreement with the experiment

The results of the comparison confirm the suitability of the mathematical models used in two-phase flow. The experience is used to model a two-phase flow in a horizontally placed 
annulus and will be used for flow in an annulus with walls treated with hydrophobic wall material and with ferromagnetic or magnetorheological fluids.

Acknowledgments: The work presented in this paper was supported by a grant GAČR 101/ 19/06666S - Research of the flow and interaction of two-component liquids with solids and external magnetic field (Výzkum proudění a interakce dvousložkových kapalin s tělesy a vnějším magnetickým polem)

The work presented in this paper was supported by a grant SGS „Research in the field of energysaving device for testing rotary hydraulic motors." SP2020/25.

\section{References}

1. J. Farník, Nestability v mezeře mezi dvěma relativně rotujícími koaxiálními válci a jejich vyšetřování. Ostrava, Disertační práce. Vysoká škola báňská - Technická univerzita Ostrava (2006)

2. M. Kozubková, J. Farnik, CD Sborník XXV. Mezinárodná Konferencia Katedier Mechaniky Tekutín A Termomechaniky, p. 7. (Modra: Slovenska technicka univerzita Bratislava) Mathematical and physical simulations of the transition from laminar to turbulent flow (2006)

3. S. Fialová, M. Kozubková, J. Jablonská, M. Havlásek, F. Pochylý, D. Šedivý, Journal bearing with non-Newtonian fluid in the area of Taylor vortices (2019) IOP Conference Series: Earth and Environmental Science, 240 (6), art. no. 062013.

4. S. Fialová, F. Pochylý, E. Malenovský, Numerical analysis and simulations of the magnetic field and hydrophobicity effect on the journal bearing dynamics. Proceedings of The Institution of Mechanical Engineers Part J-Journal of Engineering Tribology, roč. 231, č. 5, 561-571, ISSN: 1350-6501 (2017)

5. D. Šedivý, P. Ferfecki, S. Fialová, Influence of Eccentricity and Angular Velocity on Force Effects on Rotor of Magnetorheological Damper. In EPJ Web of Conferences. EPJ Web of Conferences, 555-559, ISSN: 2100-014X (2018)

6. S. Odenbach, Ferrofluids, Lecture Notes in Physics, (http:/www.springer.de/phys/) 2002

7. E. Blums, A. Cebers, M. Maiorov, Magnetic fluids. Walter de Gruyter (1997)

8. S. Odenbach, S. Thurrn, Magnetoviscous eflects in ferrofluids. Technical report, ISBN 3-540-43068-7, Springer-Verlag, Heidelberg (2002)

9. ANSYS Fluent Theory Guide, ANSYS, Inc. Release 2019 R3 Southpointe August 2019 2600 ANSYS Drive Canonsburg, PA 15317 (2019)

10. A. Davey, The growth of Taylor vortices in flow between rotating cylinders (1962) Journal of Fluid Mechanics, 14 (3), pp. 336-368. doi: 10.1017/S002211206200 1287 